\title{
Soft X-Ray Image Analysis to Detect Foreign Materials in Foods
}

\author{
Kazuo Morita, ${ }^{1}$ Yukiharu OGAWA, ${ }^{2}$ CHI N. THAI $^{3}$ and Fumihiko TANAKA ${ }^{1}$ \\ ${ }^{1}$ Kagoshima University, Faculty of Agriculture 1-21-24 Korimoto Kagoshima 890-0065, Japan \\ ${ }^{2} U S D A-A R S-W R R C$ \\ ${ }^{3}$ The University of Georgia, College of Agricultural and Environmental Sciences
}

Received March 7, 2002; Accepted January 23, 2003

\begin{abstract}
Soft X-ray images were recorded using a super metal image intensifier camera to detect foreign materials in foods, and were analyzed by a computer with an image processor. The basic characteristics of the soft X-ray image were determined using a vinyl chloride and acrylic plate. Steel screws, aluminum rivets, staples, aluminum foil, glass and plastic fragments in a loaf of bread and a hamburger steak were identified, as well as a grasshopper under cabbage leaves. In the original soft X-ray image, it was possible to detect metallic and non-metallic foreign materials more than $1 \mathrm{~mm}$ in size except for plastic fragments. However, as the original images of the bread and hamburger steak were not clear, it was not easy to detect such foreign materials visually. The unsharped masking treatment of a soft X-ray image was used to detect foreign materials in foods, and various image treatments were applied to detect the grasshopper under the cabbage leaves. The method is believed to be widely applicable for identifying the various kinds of foreign materials and foods.
\end{abstract}

Keywords: soft X-ray, image analysis, detection, food physical foreign material

Quality control of foods and agricultural products has always been one of the most difficult problems associated with handling, processing, sorting and insuring safety in the food industry. It is especially important for foods and agricultural products to have a high degree of safety, and inspections for quality control include biological, chemical and physical tests. The biological inspection is intended to detect contamination by microorganisms, the chemical inspection to detect harmful substances like agricultural chemical residues, and the physical inspection to detect physical foreign materials actually embedded in a product. In the biological and chemical inspections, most of the sampling and analytical methods are established by government standards for known hazards (Muramatsu, 1989). However, as the type of any foreign material and its location in a food product is usually unknown, there are no firm methods of physical inspection. It is especially difficult to detect foreign materials mixed in processed foods by a rapid non-destructive method as the amount and/or location of these materials are unknown. Though an inspection device for metallic foreign materials in foods is in practical use, there is a problem with its accuracy and detectability (Schatzki \& Wong, 1989; Takeuchi, 1992). It is hard to detect a soft metal like aluminum, stainless steel or a small broken piece of a metallic part of a processing machine. At the same time, there are also many nonmetallic foreign materials in foods for which no inspection method has been established (Ishikawa \& Shikiya, 1989).

Therefore, it is important to develop a physical inspection system and to establish a non-destructive inspection technique for pieces or bits of foreign materials in foods. Recently, an X-ray detection system was used in safety inspections for such materials (Morita et al., 1996; Ogawa et al., 1998). When investigating the internal condition of foods, X-rays are most effective. Al-

E-mail: morita@bio2.agri.kagoshima-u.ac.jp though there have been many research attempts at non-destructive inspection using X-rays, there has been little success in practical use (Fujii, 1989; Tollner et al., 1991). In the practical use of $\mathrm{X}$-ray, there are two common methods. One is a one dimensional method measuring the intensity of X-ray transmitted foreign materials by scintillation detectors. The other is a two dimensional method with video images using an X-ray vidicon camera. A see-through method using X-ray transmittance has been partially adopted for the physical inspection of high class ham. However, there are limits on the type and size of foreign materials and it is almost impossible to detect non-metallic materials (Kenmochi, 1992).

In this study, a continuous soft X-ray source and an image intensifier (I.I.) camera were used. The aim of the study was to obtain a basic characteristic of the soft X-ray by I.I. camera for detection of foreign materials in foods, and also to discuss the possibility of detection of foreign materials in certain foods and agricultural products.

\section{Materials and Methods}

Soft X-ray generator and detector A special modified device of SOFTEX M-80WE type (SOFTEX CO.,LTD, Ebina) was used as a soft X-ray generator in this experiment. The X-ray tube (SOFTEX I-1310 type) with a tungsten cathode and a beryllium radiation window was adopted. Unlike an X-ray through a glass radiation window, the beryllium radiation window absorbs al-most no energy in the region of the soft X-ray radiation. Under a power source condition of less than $80 \mathrm{kV}, 5.0 \mathrm{~mA}$, a continuous X-ray with a spectrum of up to $100 \mathrm{keV}$ was irradiated. Distance between the X-ray power source and the detector was $600 \mathrm{~mm}$. The peak spectrum of X-ray energy was approximately $8.4 \mathrm{keV}$ and the energy peak was in the region of the soft X-ray. A super metal image intensifier (I.I.) camera (E5844HCY-1K, 


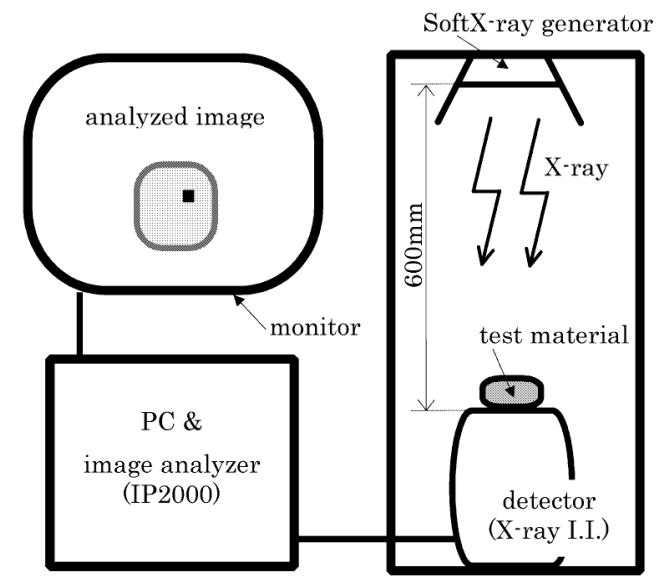

Fig. 1. Schematic diagram of soft X-ray measuring system.

TOSHIBA Co. Ltd.) was used as the soft X-ray detector in this experiment. The detecting diameter of the camera was 4 inches and it had high sensitivity in the soft X-ray region. The I.I. detector can provide transmitted X-ray image data on a monitor in real time. The CCD camera within the super metal I.I. has 400,000 pixels in its sensing area of $2 / 3$ inch diameter. The scanning frequencies are $15.73 \mathrm{kHz}$ in the horizontal direction and $59.94 \mathrm{kHz}$ in the vertical direction. There are 512 scanning lines with an interlace scanning at $2: 1$. A $1.0 \mathrm{~mm}$ thick carbon plate protected the detecting surface area of the I.I. detector, and transmitted the soft X-rays largely without absorption.

Measuring of soft X-ray IMAGE A schematic diagram of the experimental apparatus is shown in Fig. 1. To eliminate potential fogging by halation, measured objects were put directly on the sensing surface of the detector. The detector was exactly positioned under the X-ray tube using a laser beam, to eliminate any reflected X-ray from the chamber wall. The distance between the soft X-ray source and the detector was $600 \mathrm{~mm}$, and the intensity of the soft $\mathrm{X}$-ray power was fixed so that there was maximum contrast on the monitor. The soft X-ray images of foreign materials in foods were recorded and stored as 8 bit digital data in a personal computer, and were analyzed by an image processor (IP2000, Hitachi CO.). The image analysis emphasized special features of the original image by brightness values in the

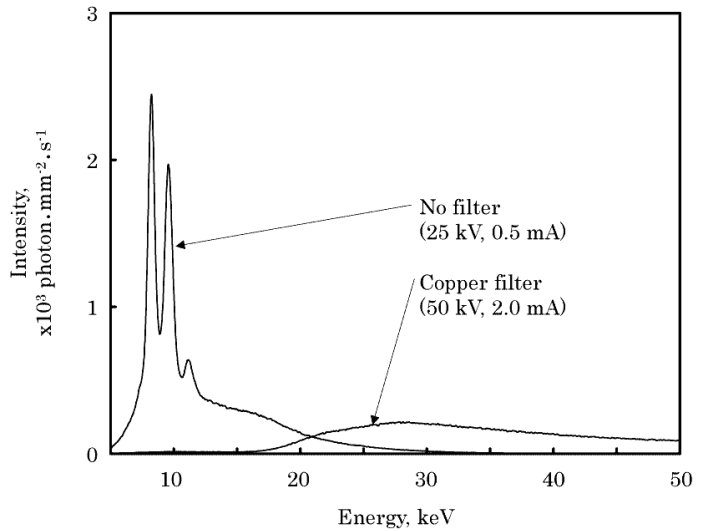

Fig. 2. X-ray spectral curves with and without filter on the radiation window.

region from 0 to 255 . The increased intensity of soft X-ray presented the image in a brighter white. There are many kinds of filtering treatments like as a derivation image with sobel filter, a low pass image with unsharped masking filter and binary image in the image analysis. In this experiment, the unsharped masking filtering treatment was used to eliminate unevenness of brightness and emphasize the edge of objects in the original image.

A loaf of bread, a hamburger steak and cabbage were selected

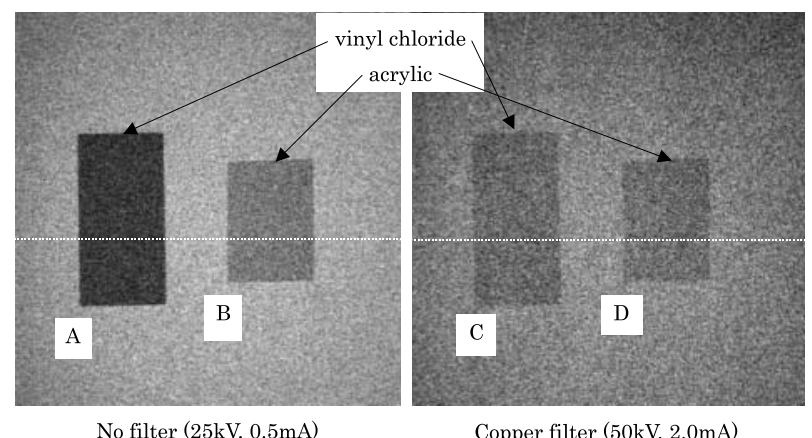

Fig. 3. Comparison of $\mathrm{X}$-ray images of vinyl chloride and acrylic plate with and without filter on the radiation window.
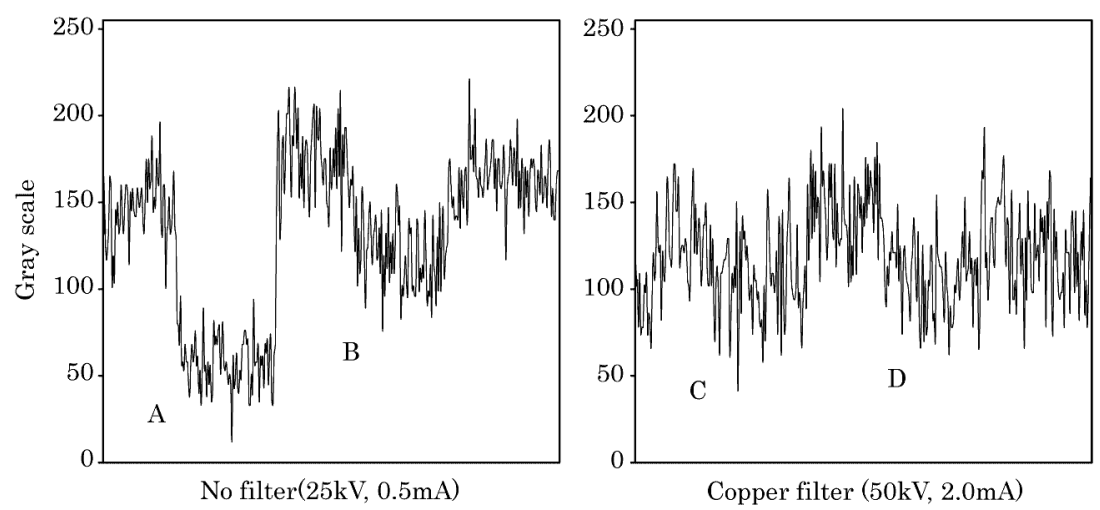

Fig. 4. Distributions of brightness of vinyl chloride and acrylic materials at white line in Fig. 3. 
as foods, steel screws, aluminum rivets, staples and aluminum foil rolled into balls of different sizes were used as metallic foreign items and glass, vinyl chloride, acrylic and grasshopper were the non-metallic foreign materials.

\section{Results and Discussion}

Basic characteristic of soft X-ray image The images were measured under different conditions of radiation to evaluate the effect of X-ray quality on the transmitted images. Figure 2 shows the continuous X-ray spectra under different conditions of radiation. The relationship was determined between the number of detected photons per minute and per square millimeter in terms of the photon energy $\mathrm{keV}$. The spectral curve at $25 \mathrm{keV}, 2.0 \mathrm{~mA}$ was measured with no filter on the beryllium window and the spectral curve at $50 \mathrm{keV}, 2.0 \mathrm{~mA}$ was measured with a copper metal filter on this window. These spectra showed different characteristics on the radiation energy level. Though the spectral

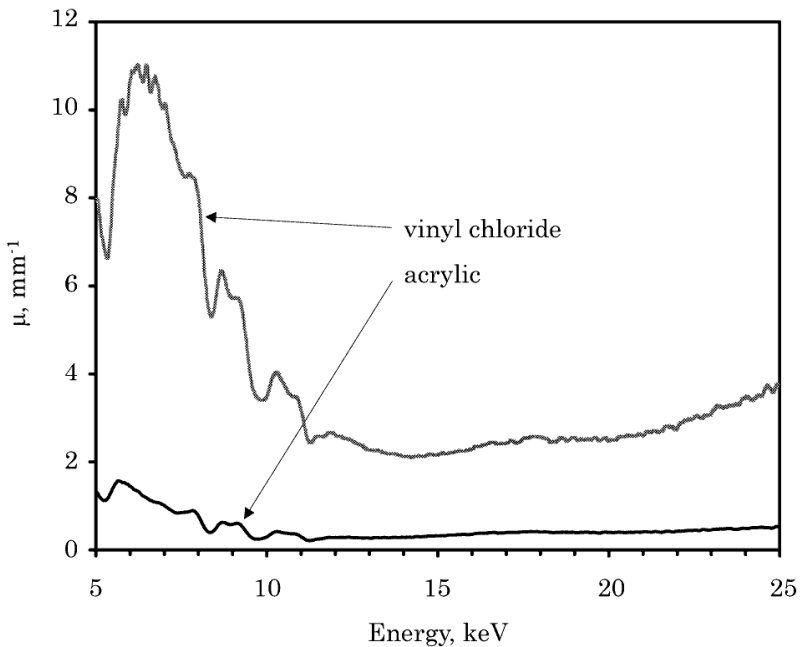

Fig. 5. Changes in $\mu$ values of acrylic and vinyl chloride materials. curve with no filter had peaks at the soft X-ray region, the peak of X-ray intensity disappeared on the spectral curve with metal filter. Figure 3 shows the transmitted $X$-ray images of acrylic $(10 \mathrm{~mm} \times 14 \mathrm{~mm} \times 2 \mathrm{~mm})$ and vinyl chloride $(10 \mathrm{~mm} \times 20 \mathrm{~mm} \times$ $0.5 \mathrm{~mm}$ ) plates at the X-ray radiation with and without filter. These test materials were directly placed on the carbon plate of the I.I. detector. Though the dark color of acrylic and vinyl chloride plates on the image with the filter was similar, there was a clear difference between these colors on the soft X-ray image. The density of vinyl chloride is higher than that of acrylic and the $\mathrm{X}$-ray absorption of the former in the soft X-ray region higher than that of the latter, making the color of vinyl chloride on the image darker than that of acrylic. Figure 4 shows a difference between brightness values of these two substances at X-ray radiation with and without the filter. The brightness values were calculated on a white line in the image of Fig. 3. As the images were not treated by a noise reduction filter, there was a great deal of

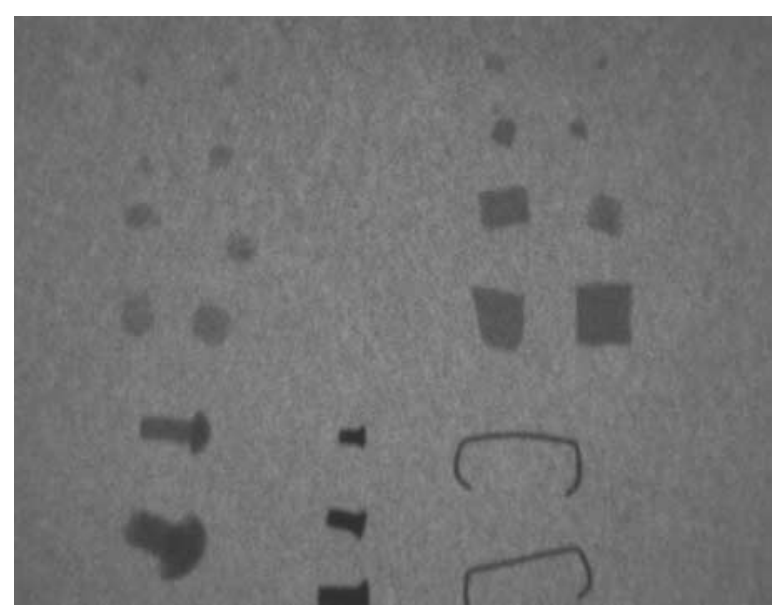

Fig. 7. Soft X-ray image of foreign materials in a loaf of bread. (40 kV, $1.0 \mathrm{~mA}$ )

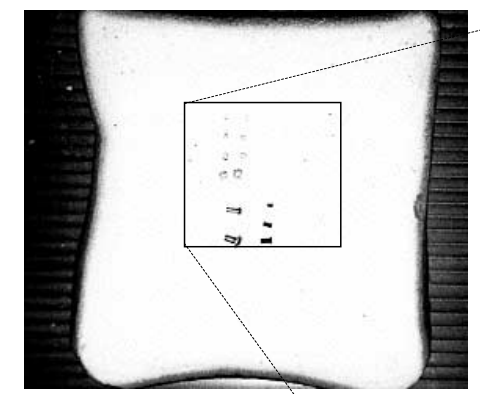

Photograph of bread

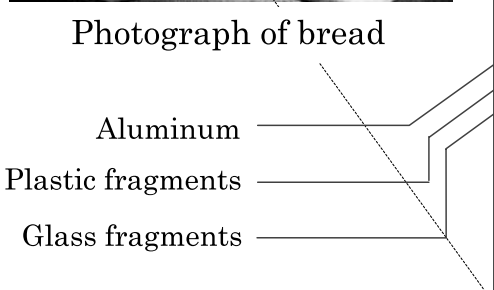

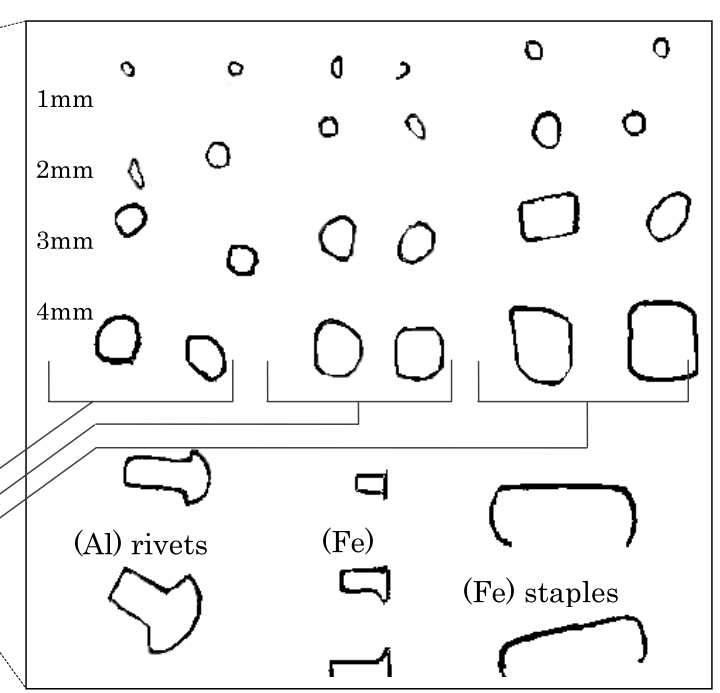

Foreign materials

Fig. 6. Various foreign materials in a loaf of bread. 
noise on the brightness curves. Compared with these brightness curves, however it was clear that the brightness value of vinyl chloride was lower than that of acrylic in the soft X-ray image. Figure 5 shows calculated values of the linear absorption coefficients, $\mu$, in the region from $5 \mathrm{keV}$ to $25 \mathrm{keV}$ for vinyl chloride and acrylic materials. The linear absorption coefficients varied greatly in the region of lower energy and the difference was especially remarkable between them at below the $15 \mathrm{keV}$ energy region. These results showed that soft X-ray images potentially can be applied to discriminate materials with different densities in non-metallic foreign materials.

Soft $X$-ray image of foreign materials in foods Figure 6 shows a schematic diagram and a photograph of various foreign materials of different sizes in a loaf of bread. Size of the foreign materials, aluminum foil, and glass and plastic fragments were processed in sizes of 1, 2, 3, $4 \mathrm{~mm}$ and $1 \mathrm{~mm}$ thickness for the purpose of discussion. The measuring condition of the soft X-ray power source was $40 \mathrm{kV}, 1.0 \mathrm{~mA}$. The original image at the location of the foreign materials is shown in Fig. 7, where outlines of these materials are not clear. Aluminum rivets, steel screws, staples and glass fragments were detected visually in the original image. But, $1 \mathrm{~mm}$ aluminum foil fragments were undetectable by this means. The plastic fragments of any size were also undetectable in the original image.

Figure 8 shows a schematic diagram and photograph of various foreign materials in a hamburger steak. The same materials and measuring conditions of the X-ray source power were used. Location of these materials in the original image is shown in Fig. 9, and as true in the image of the bread, their outlines were unclear. The aluminum rivets, steel screws, staples and glass fragments were visually detectable in the original image. Although, aluminum foil fragments of $2 \mathrm{~mm}$ were not. Again, plastic fragments of any size were not detectable. Consequently, it was possible to recognize both metallic and non-metallic foreign materials more than $1 \mathrm{~mm}$ in size except for plastic fragments. As the original images of the bread and hamburger steak were not clear, however foreign materials could not be detected visually.

The unsharped masking treatment was applied in the image analysis to emphasize the outlines of foreign materials. Though outlines in the original image were not clear, they were remarkably emphasized in the unsharped masking image. After this treatment, it was possible to automatically recognize foreign materials by computer analysis.

The computer analysis of a soft X-ray image treated by the unsharped masking was thus valid for the detection of foreign materials in foods, and is believed to be widely applicable for use in various foods.

Computer analysis of soft X-ray image Advantages provided by the filtering treatment of an image were evaluated using soft and low density materials and an insect. These are one of the most difficult combinations in which to detect foreign material. Figure 10(a) shows a soft X-ray image of a grasshopper under cabbage leaves, located in a white circle, the grasshopper was hard to find in the original image. Figure 10(b) shows the analyzed image using a sobel filter. The characteristics of both cabbage and grasshopper are emphasized in the image, but there was no difference between them. Figure 10(c) shows the analyzed image using unsharped masking: clearer than Fig. 10(b) and emphasizing the outline of grasshopper. Figure 10(d) shows the

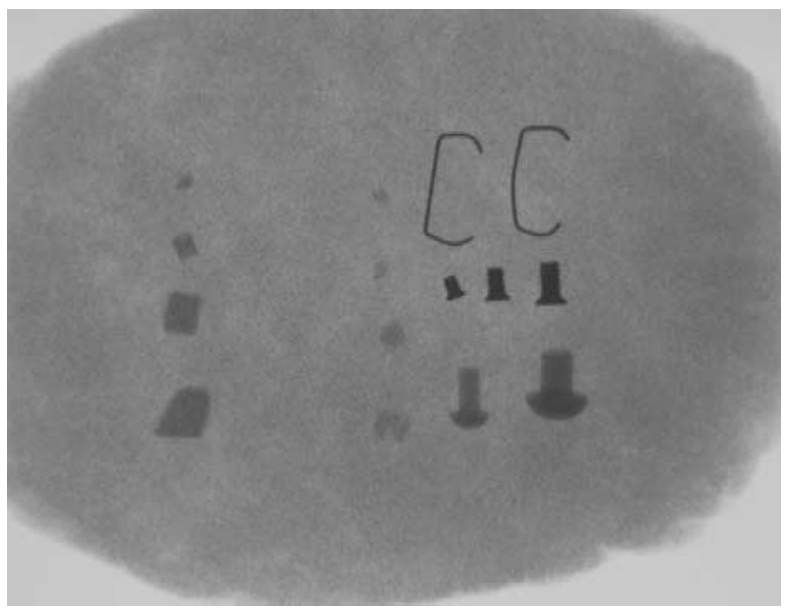

Fig. 9. Soft $\mathrm{X}$-ray image of foreign materials in a hamburger steak. (40 $\mathrm{kV}, 1.0 \mathrm{~mA}$ )

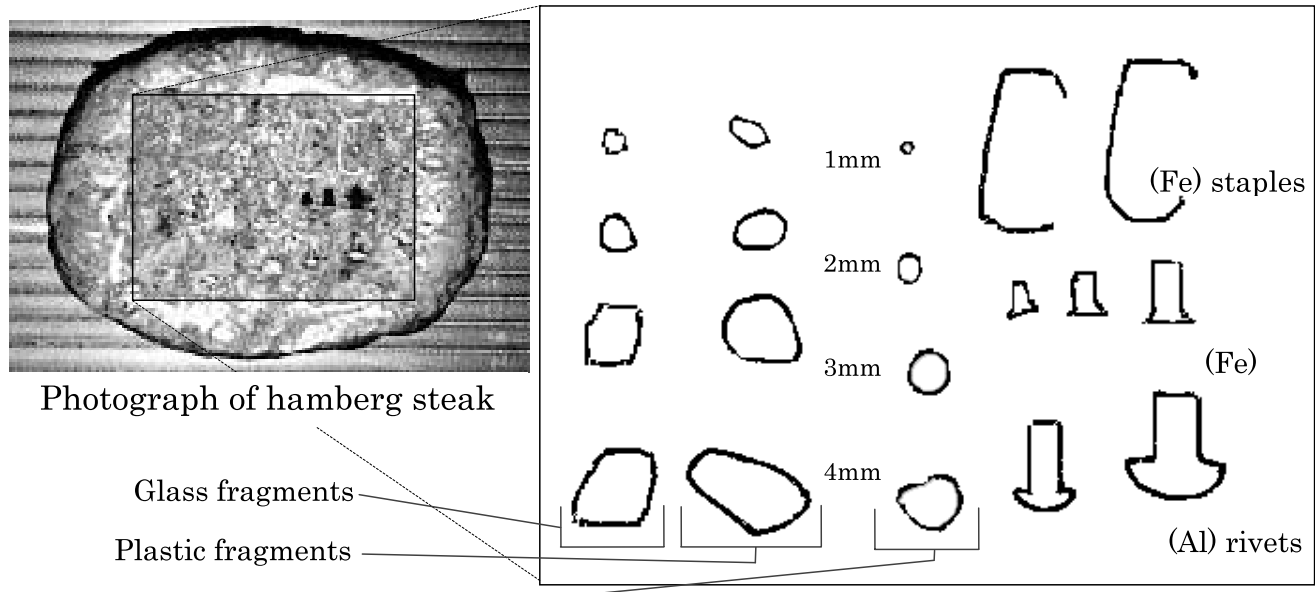

Aluminum foils $\quad$ Foreign materials

Fig. 8. Various foreign materials in a hamburger steak. 


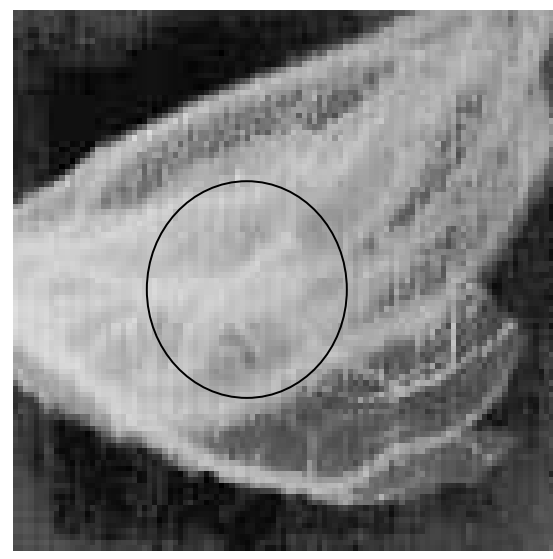

(a)

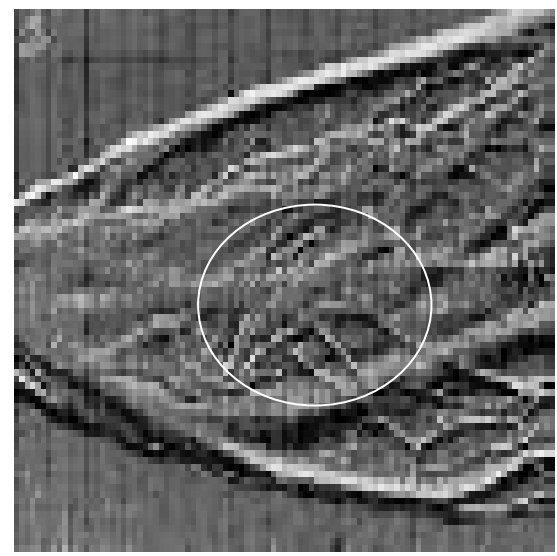

(c)

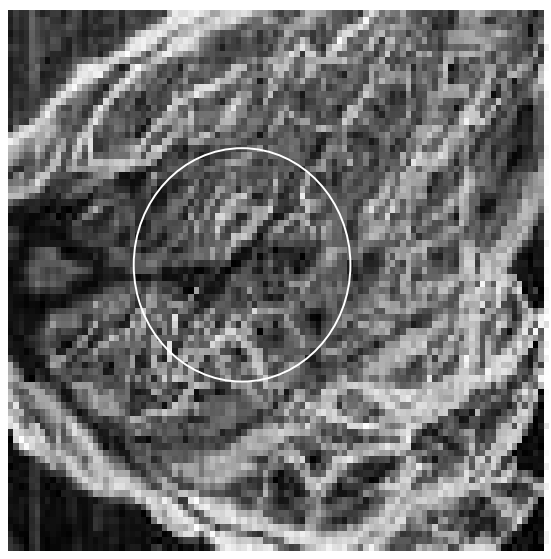

(b)

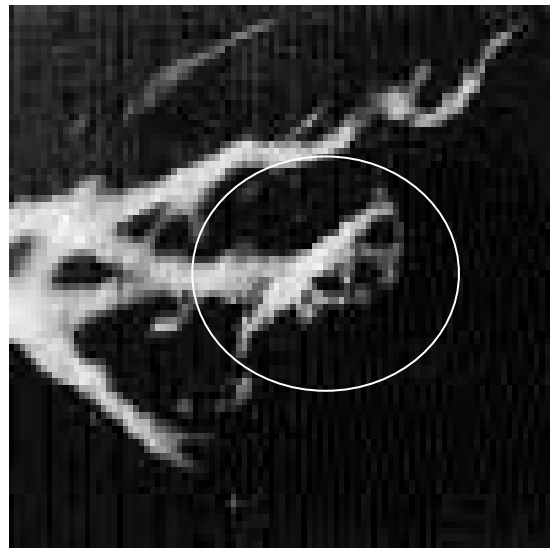

(d)

Fig. 10. (a) Image of a grasshopper in cabbage leaves. (Raw); (b), Image of a grasshopper in cabbage leaves. (Sobel filter); (c), Image of a grasshopper in cabbage leaves. (Unsharped masking) (d), Image of a grasshopper in cabbage leaves. (High pass filter)

analyzed image using a high pass filter. Most of the cabbage leaves have disappeared in the image and the grasshopper outline appears reasonably clearly.

Consequently, though the grasshopper under the leaves could not be seen in the original picture, it could be found by computer image analysis using various filters.

\section{Conclusion}

Various foreign materials were tested to learn the basic characteristics of a soft X-ray image. The images were measured with a super metal image intensifier and were analyzed using different treatments. The results are summarized as follows;

1. The difference of linear absorption coefficient between vinyl chloride and acrylic was remarkable in less than the $15 \mathrm{keV}$ energy region. These results showed that soft X-ray images potentially can be applied to discriminate materials of different density.

2. It was possible to detect metallic and non-metallic foreign materials with the size of more than $1 \mathrm{~mm}$ except for plastic fragments in the original image. However, as the original images of the bread and hamburger steak were not clear, it was not easy to detect foreign materials in them visually.

3. The unsharped masking treatment of soft X-ray image was valid for the detection of foreign materials in foods, and is believed to be widely applicable for a variety of materials and foods.

4. A grasshopper under cabbage leaves was detected by analyzing the soft X-ray image and using various filters of the computer image.

\section{References}

Fujii, M. (1989). Food inspection by X-ray. Shokuhin-kogyo, 32, 20 28 (in Japanese).

Ishikawa, S. and Shikiya. O. (1989). Detection of foreign material in food packing line. Jpn. Food Sci., 28, 61-66 (in Japanese).

Kenmochi, Y. (1992). A basic study of sensors for detecting foreign material in food. Sensor Technol., 12, 54-60 (in Japanese).

Morita, K., Tanaka, S., Ogawa, Y., Setoguchi M. and Thai, C.N. (1996). Detection of non-metallic foreign materials in food by soft X-ray system with CdTe sensor. ASAE paper. No. 966059

Muramatsu, I. (1989). Foreign material in food. Shokuhin-kogyo, 32, 20-26 (in Japanese).

Ogawa, Y., Morita, K., Tanaka, S., Setoguchi M. and Thai, C.N. (1998). Application of X-ray CT for detection of physical foreign materials in food. Transac. ASAE., 41, 157-162

Schatzki, T.F. and Wong, R.Y. (1989). Detection of submilligram inclusions of heavy metals in processed foods. Food Technol., 11, $72-76$.

Takeuchi, Y. (1992). Development of sensor for metallic foreign materials. Sensor Technol., 12, 61-64 (in Japanese).

Tollner, E.W., Harrison, R. and Murphy, C. (1991). Interpreting the pixel standard deviation statistic from an X-ray tomographic scanner. Transac. ASAE., 34, 1054-1059. 